ozone, many difficulties stand in the way of its solution; so many bodies present in the air produce such similar effects. $\mathrm{Ligh}_{t}^{t}$ as Dr. Daubeny observed, is a source of interference, and the circumstances under which the ozone seems to appear are so disconnected and variable, that I regret to be unable to report any solid progress in this direction.

When we are dealing with artificially prepared ozone, we are treating a tangible body, and we can remove nearly all obstacles to investigation; but in dealing with air the position is entirely different, and uncertainty instead of certainty meets us in every foot of our research.

Tests. - There is, up to the present time, only one recognised test for ozone-namely, the solution of the iodide of potassium and starch. Owing to its power as an oxidiser, ozone acts readily on the potassium of the test compound ; the potassium is thus separated from the iodine; the iodine, set free, com. bines with the starch, forming the iodide of starch, giving a blue colour, which is the test. Test-papers, consequently, have been used, the papers being saturated with a solution of iodide of potassium and starch. Three forms of test-papers, thus prepared, are now in use--namely, Schönbein's, Moffat's, and Lowe's. * * * Lowe has also brought out another, in which, on the recommendation of the late Dr. Dundas Thom son, fifteen grains of chalk are added to each ounce of dried starch to prevent sourness; this precaution affords great uniformity of effect. In a further research, Lowe has used what he calls a "dry powder test," using powders of dried wheatstarch combined with iodide of potassium. *** I have had sent to me within the last few days some test-papers prepared by Dr. John Day, of Geelong, Australia, who has made ozone a special object of study, and who advocates, with great ability, the views of M. Schönbein. These test-papers are remarkably sensitive, but I regret to say that I have not at present been made acquainted with the relative proportions of the ingredients used in their preparation.

In order to detect the presence of ozone in the open air, the ozone test-papers, or test-powders, are ordinarily placed in a box or ozone cage; the box is coloured dark within to avoid light, but air is freely admitted. This is the apparatus eimployed, I believe, by all the meteorologists who suppiy reports to Mr. Glaisher. The amount of ozone present is measured by the intensity of the colour, and is registered on Moffat's scale. There are objections to the ozone box, which objections have been well pointed out by Mr. John Smyth, who thinks that the velocity of air makes a distinct difference in the registration of ozone. He (Mr. Smyth) has devised an apparatus for securing greater uniformity of results.

Mr. Smyth shows, from a table of experiments made with this improved ozonometer, that there is not much difference in the quantity of sensible ozone in two masses of air of equal volume, although they may be moving at different velocities, in different directions, and under different hygrometrical conditions. He is furthermore of opinion that the ordinary test-papers do not register high enough.

The great objection to these test-papers is that discolorations are produced by other agants than ozone, particularly by iodine, the chlorides, hydrochloric acid, nitrons and nitric acid. In the atmosphere, the last-named acid, from its comparatively frequent occurrence, especially after electric storms, is a serious objection. The late $R$. D. Thomson affirmed that even carbonic acid would affect these papers when they were very sensitive. As this latter point is of importance, I tested some highly sensitive paper with carbonic acid in its nascent form and after it had been collected, and found that it occasioned a slight but evident change of colour.

It has occurred to my own mind that possibly a delicate test for ozone might be found by observing the effect produced by the electric spark when passed through rarefied ozonised air. For this purpose, a glass globe should first be filled with air that is saturated with ozone, and then be partially exhausted ; through this rarefied ozonised air an electric spark should be passed, and the colour of the spark should be noticed, and compared with the colour of the spark when passed through common air contained in the same vessel. But this experiment should be carried further by bringing the spectroscope into use. As by some, indeed by many, all gases are supposed to be the vapours of metals, it is feasible that the spectrum would give a different band or bands with rarefied ozonised air from that or those afforded by common air under similar circumstances and conditions.

(Te be concluded.)

Dr. LANzA, who formerly was in the Italian Cabinet, has just been appointed President of the Chamber of Deputies.

\section{THE SULPHITES IN TYPHOID OR ENTERIC} FEVER.

By R. S. CROSS, EsQ., M.R.C.S., L.S.A.

I BEG to lay before the readers of THE LANCET an account (abridged as much as practicable) of an outbreak of typhoid in the neighbourhood of Petersfield during the autumn of 1866 . Its history commences with the return of a family from Mentone in the first week of July, one member of which, a little boy seven or eight years old, was only just convalescent from an attack which had kept him there far into the hot season. From all I could gather, the disease was of a much more virulent kind than is usual in this country, the condition of the patient having been, in the later stages more especially, like that observed in low typhus; his father described the state of his tongue and mouth as if a coat of varnish had been applied and allowed to dry on. Almost in despair of recovery, he was started homewards, and the change appeared to produce immediate improvement; for, although the weather was intensely hot all the way, and in London, where he remained a week after his arrival in England, he got home quite recovered. On the 27th July we (Mr. Whicher and myself) were called to the lady's-maid, a thin, weak-looking woman of about forty. Her symptoms were referred to the heat and fatigue of the journey after long watching and nursing by the bedside at Mentone. At this time, the end of her first week's indisposition, I confess to have had some suspicion of the true nature of her malady - a suspicion abundantly confirmed by the appearance of the same symptoms in the lady of the house a week later, in two of the maidservants at the end of another week, then in the gardener's wife (who had been called in to assist in the kitchen), and also in the cook, butler, and others of the household-seven in all.

To go on with the history of the progress of the malady. At the end of six weeks, a woman from the parish, who had volunteered to wash the clothes in a cottage at the end of the garden, failed with it, and it was discovered afterwards that some blankets she had washed had somehow escaped disinfection. Three of her children took it in succession; then a man, his wife, and five children, in a cottage some three hundred yards from where she lived, and so it spread; in all about thirty cases occurred, from the age of three to seventy-five. Most of the cases were very severe ones, the largest number appearing to have the malady in the most intense form. One boy, about nine years of age, in particular, had to learn to talk, \&c., and seemed imbecile for some time. The aggregate duration of all the cases was ninety weeks.

The symptoms ushering in an attack were a worn expression of face, a sort of pinched look, great lassitude, pains in the head, back, and limbs, sometimes rigors, almost invariably diarrhoa, either with or without pains in the stomach and bowels, nausea or active vomiting, total loss of appetite, some thirst, sleeplessness, pulse quick, ranging between 90 and 120 ; tongue varying with the intensity of gastric symptoms; where active, would be covered with a yellow coating, edges and tip dark red; where less intense, looked sodden, large and white; always tremulous; and this condition, together with the quick pulse and worn look, often existed many days, and almost weeks, before the patient complained very much, or gave up and took to bed. Nothing varied so much as the character and duration of the premonitory stage.

In one patient, the lady of the house, there was positively no warning. She became paler than usual, and so rapidly weaker and weaker, that on the third night of my attendance upon her, with a perfectly clean tongue, without diarrhoea or sickness, and in the entire absence of any dread of the disease, she was only saved from sinking by the unsparing and sustained use of brandy (two-thirds of a bottle this night), and such was the character of her illness throughout.

In another case, that of a stout plethoric girl of eighteen, the malady was ushered in suddenly by symptoms of most active biliary disorder, soon followed by the wildest delirium requiring restraint for many days to keep her in bed even; both lung and intestinal complication following in the progress of the case, which was very long and doubtful. In many cases, the tongue was for days together dry and hard 
like toast, abdomen tympanitic and tender, and the lungs the seat of frequently recurring congestion, engorgement and solidification, or whatever term may best describe a condition in which a very large portion of either one or both of these organs is often perfectly useless, if not worse; sore mouth, bleeding from the fauces, tongue, and gums, loss of almost the entire mucous covering, with deep ulcerated fissures, constituted a dreadful aggravation of suffering in numerous instances. The treatment up to August 19th had been such as is usually followed. In every case where indicated, a single dose of calomel, or an emetic, was administered at the outset, and always with advantage. At this period, not being much impressed with a belief in the power of any known mode of ordinary treatment to do much in combating the disease, and not daring with six or eight people about me as good as dying to adopt the do-nothing plan, I determined to try the sulphites, the more especially after a careful perusal of the very able and exhaustive article from Dr. de Ricei in Braithwaite's Retrospect, vol. 49. I prescribed the sulphite of soda in scruple doses every four hours in water, adding for the most part quinine in grain doses as the purely gastric symptoms disappeared, and were followed by the toast tongue, and other signs of greater encroachment on the vital powers. There was a liberal, very liberal, allowance of wine, brandy, and beef-tea given, and the quantity taken every twenty-four hours by most of the sufferers was truly astonishing. We had two good nurses from the Institution in London, besides other help. It was just pouring down the three things before named day and night to keep the flickering lamp of life alight; and, without a single exception, just in proportion as the hand was stayed from any cause, either accidentally in the nursing so many, or in obedience to an order from me, based on some fear or suspicion of over-stimulation, so did the jeopardy of the patient immediately increase, and time and again it seemed hopeless that the fuller use of the brandy bottle could be in time to snatch the sufferer from the very jaws of death. Some took a bottle of wine"every twenty-four hours for three!weeks at a stretch; and the way in which this was freely placed at my disposal by the fearless, intelligent master of the house, together with champagne, ice, grapes, and everything that could be thought of, elicited my admiration, as much as his tact, discrimination, and appreciation of symptoms at the bedside by day and night, deserved my warmest thanks, as the greatest possible help in the management of such a visitation.

In the treatment of the diarrhoa, or, more properly speaking, the repeated loose action of the bowels, which was such an invariably prominent accompaniment, I did not adopt either the astringent or castor-oil plan in their entirety. Whether or not the increased action in question be due to the elimination by the glandular and mucous apparatus of the intestinal tract of a morbid poison from the blood, or the disease itself consist in a primary affection of these structures of a specific kind, true it is, practically, that in every case there is, to begin with, a most serious invasion upon the powers of life; and the cardinal point in treatment is not to allow the patient to either do or suffer anything whereby the jeopardy arising from this great exhaustion might be increased, at the same time avoiding a too active interference--an interference, I mean, amounting virtually to arrest of what, up to a certain point, may be a salutary process, and can be after all but one of many circum. stances adverse to life. I prescribed now and then an astringent mixture, containing a small quantity of laudanum-in fact, the nurses were allowed to give a certain quantity of it to restrain a little when the actions were very frequent, particularly when occurring immediately after taking support of any kind. I am no stranger to the value of a dose of castor oil under some peculiar conditions of distended abdomen, \&c.; but it is so nice a point that I verily believe a patient's life often hinges upon the fine discrimination as to its immediate applicability to the case under observation. Turpentine stupes were always going either to the chest or abdomen; and they seemed as valuable in one place as the other, never failing to relieve alike the over-burdened and sometimes painful lung, and the distended, flatulent, and uneasy belly. The subject is practically inexhaustible; but I will bring this part of my remarks to a close by saying that there was, with the exception of the patient verging on eighty, who had the fever after nursing her daughter and family for weeks, and who finally succumbed to bed-sores, no death.

I also used the sulphite as a prophylactic; a bottle of mixture was established in the house, and given in scruple doses three times a day to five people. Of these five one was the constant attendant on the lady, two were in the kitchen, another was a keeper, who came in and out, and the butler.
The attendant, though in the sick room night and day for weeks, never ailed anything. Of the two in the kitchen, one never ailed; the other, the cook, who was always running in and out the sick rooms, had all the symptoms of the malady, - the worn look, the sickness and purging, loss of appetite, quick pulse, characteristic tongue, \&c., but never gave in ; used to lie down and get up, but never was absent from her post a day. The keeper, the husband of the woman who took it by washing, was less affected, and also kept his post. The butler hung in the balance for a fortnight or three weeks, then took to his bed, and was among the worst.

Much as I believe it, I am hardly in a position to assert as much, but my experience powerfully endorses the opinion expressed by $M$. de Ricci in the British and Foreign MedicoChirurgical Review, April, 1867, p. 523, wherein he predicts. "that eventually the treatment of zymotic diseases by the administration of the sulphites will be as fally recognised as that of cinchona."

All I trust is, that after a careful perusal of this paper, I shall not be thought to have intruded these very imperfect and crude observations (recorded necessarily in the midst of much to divert) altogether uselessly upon the notice of my professional brethren, to many of whom an epportunity for making a similar trial may not only never have occurred, but be long wanting.

To proceed. On July 30 th (current year) I was requested to. see Miss —, aged twenty, residing at a distance of eight miles in a country house, the inmates of which were, and always had been, particularly healthy. I was told it was the third day of her being in bed, and the history of the case pointed simply to more manifestation of fatigue of late, in the face, as it chanced, of more than usual call and inducement for exertion. A dose of oil had been given three days prior to my visit, and had done well. On entering her room, the position in bed, her manner and appearance, voice, and cxpression of face, all reminded one of the impression of fever. The pulse was weak and quick, over 100 ; tongue sticky and coated; breath offensive; no appetite; bowels uneasy, somewhat fuller and more tense than natural; no action since that from the oil. She was unable to sit up in bed without help, or to remain long so without faintness. Ordered to take ten grains of sulphite of soda every four hours, and pills at night containing a mild dose of mercury with chalk, rhubarb, ipecacuanha, and hyoscyamus. To have beef-tea; claret-and-water to be given freely as a drink.

July 3lst (next day).-Condition essentially the same, but weaker; pulse quicker. Ordered claret without water. Pills had acted twice; feared to repeat them in the face of so much exhaustion. To continue the sulphite.

Aug. 1st.-Much worse in every respect; pulse almost too quick to count, and the tendons of the muscles of the forearm: were felt to twitch constantly under the finger, strongly enough to be seen also in other muscles; abdomen more distended, and slightly tender on pressure. Ordered turpentine stupe, tablespoonful of brandy every hour in arrowroot, beef-tea, \&c. Sulphite to be continued. Bowels rather relaxed.

2nd.-Much the same; in danger of sinking from hour to hour. Brandy increased to six drachms every two hours and the other two; two ounces of port wine to be given; beef-tea; sulphite continued. Bowels the same; slight cough. Remained the night.

With very little alteration, this condition prevailed up to the 9th, when, the bowels acting rather more frequently than I liked-the ingesta quickly passing through, almost unchanged, -I ordered a pill at night, containing half a grain of opinm, half a drop of creasote, and one grain of camphor.

The chest symptoms had fairly set in, there being complete dulness on percussion and entire absence of respiratory murmur over the lower half of left lung; with slight pain, and that coarseness of breath-sound on the right side which I have noticed to be the sure forerunner of that alteration in the lung. tissue which occurs in connexion with the disease in question, as well as not unfrequently constituting a separate and distinct malady. Both sides of her chest were thus affected, and the turpentine stupes were applied again and again, always with marked benefit. The abdominal symptoms had appeared to decline from the first application of turpentine, and especially from the date of appearance of the chest symptoms; nor had the tongue been dry and baked for more than twenty-four hours before time sufficed for getting the system under the influence of the maximum quantity of stimulus. The creasoteand-opium pills were given occasionally, eight in all.

At this period-the end of the fortnight-an ounce of the sulphite in all had been taken. And with regard to the time 
of its continued exhibition, I am disposed to think it should be dependent on the period of the malady at which it is begun, and the power it manifests to control the progress of symptoms. M. de Ricci says that "the want of success which has sometimes been observed in the treatment of zymotic diseases by the alkaline and earthy sulphites is attributable to the fact that these remedies have not been administered early enough. If the treatment is too long delayed, the blood becomes so loaded with poison and deteriorated in quality as to be no longer able to perform its normal function; and then the sul. phites are of no more service than any other remedies, because they cannot restore to life the dead blood-corpuscles. The sulphites should therefore be administered early, while still a large portion of the blood is in a healthy state, and capable not only of carrying on life, but of throwing off what has been rendered inert by the presence of the sulphuric acid." M. de Ricei attributes another source of failure to "the administration of hyposulphite of soda instead of the sulphites, and especially the sulphite of magnesia. The hyposulphite of soda is less efficacious than the sulphites, because, in the former, the greater part of the acid becomes oxidised in its passage through the animal economy, and appears in the urine as a sulphate; because, being a salt of hyposulphurous acid, it is a less active antagonistic; and because it often canses a troublesome diar rhcea: while the sulphites of soda and magnesia never produce such effects." And so on in the article in question.

To return to my patient. I discontinued, then, the sulphite at the end of the fortnight, and ordered a mixture of chlorate of potash, ammonia, cinchona, and chloric ether on the 14th, which was continued to the 20th. Somewhat later than this, the pulse having improved, and finding her at my visit somewhat flushed, feverish, and excited, I ventured to diminish one half of her stimulants; but I was only too glad to put it on again the next day, and lost three days most certainly by the experiment. And it appears to me most important to be very cautious about reducing the allowance of wine \&c., allowing the patient to sit up in bed or make any exertion, or to take solids too soon. Castor oil was given twice: the first time at the end of a month from the time of her attack; and she was able to be down stairs and take carriage exercise at the end of six weeks, from which point her recovery was rapid and complete.

The other case I was called to on Sept. 15th. I will be brief with it. A young man of twenty, stout, florid complexion, was a draper's assistant at a neighbouring seaside town. Had been ill there three days. He was prescribed for, the medicine purging him freely. Had been ill a week altogether at the time of my being called to him after his removal home. He had all the symptoms of a severe attack of the fever in question. The lung-complication was thus early extreme on both sides. He became breathless on the slightest exertion, and almost fainted on my attempting to sit him up to examine his chest posteriorly. Ordered turpentine stupes, sulphite, beef-tea, wine, \&c. The case went on to the $27 \mathrm{th}$, when he got rather suddenly much worse, I believe from neglect in the administration of support and stimulus. He was restless, with wandering delirium, dilated pupils, confusion about the head, \&c. The mouth and fauces were covered with a thick coat of aphthous exudation. These parts were sponged out well with a solution of the biborate of soda, the hair was re noved, and a mustard plaster applied to the nape of the neck; and he was ordered to take in the course of the next twentyfour hours a bottle of wine, and a tablespoonful of brandy occasionally. The next day he was much better in every respect, and continued to mend from that time, making an excel lent recovery, and passing out of our hands well on the 16 th of October : just one month ill. He took in all two ounces of the sulphite.

I could give a later case, in which the very early use of the remedy seemed to cut short the malady, and reduced the term of indisposition to but a fortnight altogether; but I forbear, having already, I fear, trespassed at too great length.

I should have mentioned that in all cases I had Burnett's solution kept in the utensils used by the invalids, these being removed immediately after use. Chloride of lime was kept going under the beds, and about the rooms and passages. I insisted on as much air as could be got, without placing the patient in a thorough draught. One of the quickest and best recoveries of the very bad cases occurred in the gardener's wife, whose bed was so situated (in a small room without a fireplace) that the wind blew in at the window upon her day and night; and another, the boy before alluded to, the window of whose room was clean gone, a sack hung up doing duty for one, but which I often found down, admitting the wind, and sometimes the rain, on to the little fellow's bed, which was shared by a brother ill of the same disease.

I would observe, in conclusion, that to many the foregoing remarks may read more like an advocacy of the ultra-stimulating plan of treatment, than that by the sulphites. To such I would say, is it not necessary in all maladies to maintain the powers of life in such a way as shall give time and opportunity for remedies to take effect? and is not the degree of energy with which this support is supplied in direct proportion to the patient's need? The quantity of stimulus must not be measured by spoonfuls, but by the observation of how much is required to keep the patient alive, and in a likely condition to be benefited by all other adjuvants, chemical and otherwise.

Petersfield, Dec. 1867.

\section{NOTE ON THE LATE NEW ZEALAND WAR.}

By HENRY SLADE, Esq., SurgeoN R.N.

(Concluded from page 45.)

THE cases of four men of the Naval Brigade who recovered from dangerous wounds of the chest and abdomen, recorded by Inspector-General Mouat in his paper, were treated at first on board H.M.S. Miranda, and sent subsequently to the Military Hospital, as the navy possesses no medical establishment in New Zealand. Two of these were cases of wounds of the lung, in which the ball had lodged in the chest, in men aged twenty-seven and twenty-five years respectively. The first also had an arm fractured by a blow which knocked him down in the pah where he was wounded. The second, besides being wounded in the lung, had a ball through the anterior wall of the pelvis from the left hip to the pubes, and a superficial wound of the hand. He lay in the pah all night after being wounded, amongst the Maoris who occupied it. $\mathrm{He}$ lost so much blood that he was unable to move, but remained perfectly sensible, and gave a harrowing description of being searched more than once in the night by the natives, who robbed him of his arms, and left him for dead amongst the dead and dying. The hæmorrhage during the night and exposure to the cold had blanched his skin, and gave him the waxy hue of a chlorotic girl. Probably this very exposure and quietude saved his life, from the opportunity it afforded for the coagulation of the blood in the lacerated vessels.

The difference between the earlier symptoms in these two cases of gunshot wounds of the chest in the first weeks of their treatment was very instructive. The first man did not cough up blood, and the emphysema was circumscribed, showing that the bullet had probably wounded but not entered the lung; while the severe paroxysms of pain referred to the region of the heart, and accompanied by sudden collapse, which he sometimes suffered from, led to the belief that the ball moved in the pleura. In the second case, a great deal of blood was coughed up, and the emphysema became general over the chest, neck, and face. In neither case was the emphysema supposed to be due to fractured rib.

The third case was that of a man wounded in the sacrum by a musket-bullet in the attack on the same pah. The bullet passed through the left side of the bone, and lodged in the pelvis, where ineffectual attempts were made to reach it by enlarging the opening while the patient was under chloroform. Its site, however, could never be determined. At one time, from the intense pain and redness about the right trochanter and thigh, it was thought to have passed out of the pelvis by the sciatic notch, and to be lodged near the hip-joint, pressing on the sciatic nerve. The patient himself thought the ball had lodged about the middle of Poupart's ligament, and here an abscess afterwards formed, but the ball was not discharged from it. After " 470 days," according to the Military Hospital returns, the ball was supposed to be half way down the thigh, where, in certain positions of the leg, the patient believed he could feel it; the health of the man being represented to be very good, and he was able to walk about. This case affords a curious instance of the course taken by a ball without any important viscus being wounded; by the ball itself, or the numerous splinters which were torn from the sacrum, and afterwards either removed by operation or discharged from 